\title{
Two-component mixture of charged particles confined in a channel: melting
}

\author{
W. P. Ferreira,, G. A. Farias, ${ }^{1, \oplus}$ and F. M. Peeters ${ }^{1,2, \text { t }}$ \\ ${ }^{1}$ Departamento de Física, Universidade Federal do Ceará, \\ Caixa Postal 6030, Campus do Pici, 60455-760 Fortaleza, Ceará, Brazil \\ ${ }^{2}$ Department of Physics, University of Antwerp, Groenenborgerlaan 171, B-2020 Antwerpen, Belgium
}

(Dated: November 9, 2018)

\begin{abstract}
The melting of a binary system of charged particles confined in a quasi-one-dimensional parabolic channel is studied through Monte Carlo simulations. At zero temperature the particles are ordered in parallel chains. The melting is anisotropic and different melting temperatures are obtained according to the spatial direction, and the different types of particles present in the system. Melting is very different for the single-, two- and four-chain configurations. A temperature induced structural phase transition is found between two different four chain ordered states which is absent in the monodisperse system. In the mixed regime, where the two types of particles are only slightly different, melting is almost isotropic and a thermally induced homogeneous distribution of the distinct types of charges is observed.
\end{abstract}

PACS numbers: 64.70.dg, 61.46.Hk, 64.70.dj

\section{INTRODUCTION}

Recently, there has been a renewed interest in the study of the physical properties of multi-component systems [1 3]. The presence of particles with distinct physical properties (e.g. size, charge, mass) introduces a competition between different scales, which is the reason for the richer phenomenology in such systems [2]. The simplest multi-component system is a binary mixture of two types of particles which, as compared to a mono-disperse system [4 10], has been shown to exhibit a very rich phase diagram. Such binary mixtures have been studied in several different experimental setups such as ion traps [11, 12], magnetized disks floating at a liquid-air interface in an external magnetic field [13], dusty plasmas [14] and colloidal suspensions [15].

Under specific conditions of density and temperature a system of interacting particles will solidify into an ordered arrangement forming a crystal structure. When the inter-particle potential is purely repulsive this is often referred to as a Wigner crystal, in reference to the crystallization of the electron gas predicted by Eugene Wigner [16], and observed experimentally for the first time in a 2D system of electrons on the surface of liquid helium by Grimes and Adams [17]. In three-dimensional case (3D), a first experimental investigation of classical plasma crystals can be found in Ref. [18]. Such an ordered phase appears in several non-electronic systems, and has also been experimentally observed, e.g. in ion traps 11, 12], colloidal systems [19, 20], and dusty plasmas [21 23]. Besides the single-component system, which has been widely studied in the last years, crystallization may also be observed in multi-component systems of in-

\footnotetext{
*Electronic address: wandemberg@fisica.ufc.br

$\dagger$ Electronic address: gil@fisica.ufc.br

${ }^{\ddagger}$ Electronic address: francois.peeters@ua.ac.be
}

teracting particles [1, 2], and particularly in binary systems [7]. Recently, a large number of different equilibrium configurations, which depend on the relative fraction of the different types of particles, was found in a two-dimensional (2D) system of interacting dipoles [6].

In the case of a quasi-one-dimensional (Q1D) system of repulsive particles several recent experimental [22 25], analytical and numerical [9, 10, 26] studies showed interesting physical properties and promising perspectives for practical applications [27]. Systematic studies of Q1D systems of interacting particles confined in parabolic 9 and hardwall [26, 28] channels revealed many different phases of chain-like structures, and even poorly ordered configurations. The latter depends on the nature of the confinement potential and is favored by hard wall-type of confinement.

An interesting and very important physical phenomenon is melting. In the $2 \mathrm{D}$ case, the transition from a solid phase to a complete isotropic liquid phase can be characterized by a two-step process with an intermediate hexatic phase [29 31]. However, such a melting scenario is not unique and depends on the considered system, i.e. the inter-particle interaction, and its dimensionality. Furthermore, previous papers showed also that the melting of $2 \mathrm{D}$ clusters is non-universal [19, 32, 33].

The specific structural arrangement of the particles in confined systems has a very important effect on the nature and specific process of melting. In the case of $2 \mathrm{D}$ mono- and bi-disperse confined clusters, structural properties of the ordered configuration were found to strongly influence the melting [8, 33]. E.g., the melting temperature in small clusters of charged particles was found to be a nonuniform increasing function of the strength of the binding of the cluster [34]. It was demonstrated in Ref. 34] that the symmetry of the configuration is one of the dominant factors that determines the melting temperature. In the case of small confined binary clusters, we showed recently that thermally induced structural phase transitions can occur before melting takes place. In ad- 
dition, a remarkable temperature induced spatial phase separation was found [8].

In the present paper we use Monte Carlo simulations to study the melting of a binary system of classical charged particles confined in a quasi-one-dimensional parabolic channel. The structure and normal mode spectrum of such a system were recently addressed by us in Ref. [10]. A very rich set of chain-like structures with, in some cases, an intrinsic spatial separation of the distinct types of particles was predicted. A density dependent bandgap in the phonon spectrum was found, and a softening of the normal mode frequencies characteristic of a continuous structural transition from the mixed single-chain to the segregated two-chain regime. In the present analysis we limit ourselves to an equal density of the two types of particles which are assumed to have the same mass in order to reduce the number of parameters. Most of the results are given for the representative case where the charge of one type of particle is twice the charge of the other one. Special attention will be given to the particular case where the charges are almost equal. In this regime at zero temperature, an asymmetrical distribution of charges over the chains was found previously [10], while here we find that temperature leads to a mixing of the particles and finally a homogeneous distribution of particles.

The paper is organized as follows. In Sec. II, we describe the model and the procedure used to calculate the melting temperature. In Sec. III the results for the melting of different ordered structures corresponding to different regimes are discussed. Our conclusions are given in Sec. IV.

\section{MODEL}

We study a two-dimensional binary cluster consisting of an equal number of particles with distinct charges $q_{a}$ and $q_{b}$, which are allowed to move in the $x-y$ plane. The charged particles interact through a repulsive DebyeHückel (or Yukawa) potential $\exp (-r / \lambda) / r$, are free to move in the $x$ direction, and are confined by an external one-dimensional parabolic potential which limits the motion of the particles in the $y$ direction. A parabolic confinement potential was realized in Ref. [22] for a quasione-dimensional system of dusty plasma and in Ref. [35] for the case of colloidal particles confined into a droplet region. Such a Yukawa inter-particle potential describes, e.g. charged colloidal particles in a liquid environment, and dusty particles in a plasma. The screening length $\lambda$ can be varied by changing the density of the counterions (plasma) in the colloidal (dusty plasma) system. In the case of a dusty plasma the lateral parabolic confinement potential was recently realized by structuring the bottom electrode into a channel [36]. It was demonstrated that the electric field induced potential is parabolic whose strength depends on the width of the channel and the electric field strength. For the charged colloidal system the colloids are e.g. confined between two glass plates which can be structured into a channel 24 26]. In this case the confinement potential can be represented by a hard wall potential. It was shown recently [37] that by curving the bottom of the channel it is possible to induce a parabolic confinement potential for the colloids where the confinement frequency can be varied by changing the curvature.

The potential energy of the system is given by

$$
\begin{aligned}
H= & \sum_{i} \frac{1}{2} m \omega_{0}^{2} y_{i}^{2}+\frac{q_{a} q_{b}}{\epsilon} \sum_{m} \sum_{n} \frac{\exp \left(-\left|\mathbf{r}_{m}^{(a)}-\mathbf{r}_{n}^{(b)}\right| / \lambda\right)}{\left|\mathbf{r}_{m}^{(a)}-\mathbf{r}_{n}^{(b)}\right|} \\
& +\frac{q_{a}^{2}}{\epsilon} \sum_{i>j} \frac{\exp \left(-\left|\mathbf{r}_{i}^{(a)}-\mathbf{r}_{j}^{(a)}\right| / \lambda\right)}{\left|\mathbf{r}_{i}^{(a)}-\mathbf{r}_{j}^{(a)}\right|} \\
& +\frac{q_{b}^{2}}{\epsilon} \sum_{k>l} \frac{\exp \left(-\left|\mathbf{r}_{k}^{(b)}-\mathbf{r}_{l}^{(b)}\right| / \lambda\right)}{\left|\mathbf{r}_{k}^{(b)}-\mathbf{r}_{l}^{(b)}\right|},
\end{aligned}
$$

where $\epsilon$ is the dielectric constant of the medium the particles are moving in, $\lambda$ is the Debye screening length, and $\mathbf{r}_{i}^{(A)}=\left(x_{i}, y_{i}\right)$ is the distance of the $i^{\text {th }}$ particle of type $A$. In order to reveal the important independent parameters of the system, it is convenient to write the energy and the distances in units of $E_{0}=\left(m \omega_{0}^{2} q_{a}^{4} / 2 \epsilon^{2}\right)^{1 / 3}$ and $r_{0}=\left(2 q_{a}^{2} / m \epsilon \omega_{0}^{2}\right)^{1 / 3}$, respectively, and define the quantity $\alpha=q_{b} / q_{a}$ (in the following we express $q_{a}$ and $q_{b}$ in units of $e$, the elementary charge), and the screening parameter $\kappa=r_{0} / \lambda$. In so doing, the expression for the potential energy is reduced to

$$
\begin{aligned}
H= & \sum_{i} y_{i}^{2}+\alpha \sum_{m} \sum_{n} \frac{\exp \left(-\kappa\left|\mathbf{r}_{m}^{(a)}-\mathbf{r}_{n}^{(b)}\right|\right)}{\left|\mathbf{r}_{m}^{(a)}-\mathbf{r}_{n}^{(b)}\right|} \\
& +\sum_{i>j} \frac{\exp \left(-\kappa\left|\mathbf{r}_{i}^{(a)}-\mathbf{r}_{j}^{(a)}\right|\right)}{\left|\mathbf{r}_{i}^{(a)}-\mathbf{r}_{j}^{(a)}\right|} \\
& +\alpha^{2} \sum_{k>l} \frac{\exp \left(-\kappa\left|\mathbf{r}_{k}^{(b)}-\mathbf{r}_{l}^{(b)}\right|\right)}{\left|\mathbf{r}_{k}^{(b)}-\mathbf{r}_{l}^{(b)}\right|}
\end{aligned}
$$

and the state of the system is determined now by the ratio between the charges $\alpha$, the number of particles per computational unit cell (which is related to the linear density), and the dimensionless screening length $\kappa$. The dimensionless linear density $n$ is defined here as the ratio between the number of particles in the unit cell and the length of the unit cell in the unconfined $x$ direction. Periodic boundary conditions were considered in the $x$ direction in order to mimic an infinite system. The largest size for the simulation box that we considered along the $x$ direction was $L=50$ unit cells ( $\mathrm{N}=400$ particles), and the results were the same as the ones found with $L=25(\mathrm{~N}=200$ particles $)$ and $L=12.5(\mathrm{~N}=100$ particles) unit cells, indicating that the results do not depend on this length. The temperature is expressed in units of $T_{0}=E_{0} / k_{B}$, where $k_{B}$ is the Boltzmann constant. From 

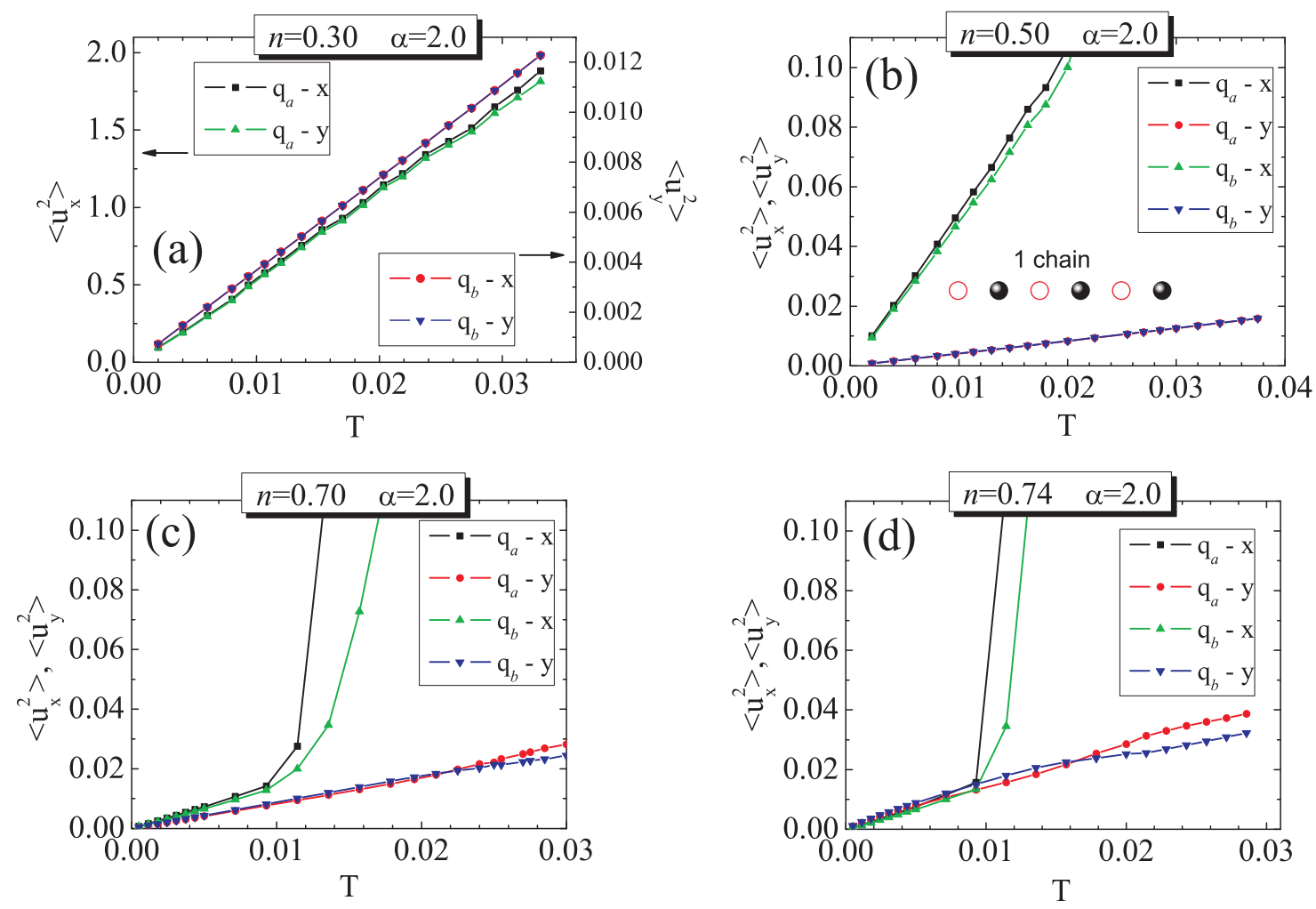

FIG. 1: (Color ) The relative displacements squared $\left\langle u_{x}^{2}\right\rangle$ and $\left\langle u_{y}^{2}\right\rangle$ for the distinct types of charges $\left(q_{a}=1, q_{b}=2\right)$ as a function of temperature for different densities (a) $n=0.30$, (b) $n=0.50$, (c) $n=0.70$, and (d) $n=0.74$ in the single-chain regime. The ground state configuration for the single-chain regime is shown as inset in (b).

now on, all quantities presented in the manuscript are in dimensionless units. The charges, in particular, is in units of the elementary charge $e$.

The minimum energy configurations and the normal modes of this system were systematically analyzed recently in Ref. [10]. In the present work we employ Monte Carlo (MC) simulations to investigate the thermal dependence of the minimum energy arrangements. Starting from a small temperature, typically $5 \times 10^{-4} T_{0}$, the system is equilibrated during typically $10^{6} \mathrm{MC}$ steps. The properties of interest are then measured and averaged over the next $10^{6} \mathrm{MC}$ steps. This procedure was typically repeated 15-20 times and the quantities of interest averaged over those 15-20 runs. The temperature was increased in steps of the order $\Delta T \approx 10^{-3}-10^{-2}$, and the previous process was repeated.

It is well-known that in $2 \mathrm{D}$ and $1 \mathrm{D}$ the root mean square deviation of the particles from their average lattice position diverge with the size of the system when the temperature is nonzero. This feature was interpreted as preventing long-range order in $2 \mathrm{D}$ and $1 \mathrm{D}$. In the meantime we know that 2D and $1 \mathrm{D}$ ordering is possible even at nonzero temperature. In order to characterize melting, we consider therefore a modified Lindemann parameter [9, 33], $L_{p}=\left\langle u^{2}\right\rangle / d_{r}^{2}$, where $\left\langle u^{2}\right\rangle$ is the difference be- tween the mean square displacement of neighboring particles from the respective equilibrium positions $\vec{r}_{0}$, and $d_{r}$ is the mean interparticle distance which is defined below. The quantity $\left\langle u^{2}\right\rangle$ is given by the expression

$$
\left\langle u^{2}\right\rangle \equiv \frac{1}{N}\left\langle\sum_{i=1}^{N} \frac{1}{N_{n b}} \sum_{j=1}^{N_{n b}}\left[\left(\vec{r}_{i}-\vec{r}_{0 i}\right)-\left(\vec{r}_{j}-\vec{r}_{0 j}\right)\right]^{2}\right\rangle,
$$

where \langle\rangle means the average over the MC steps, $N$ is the total number of particles in our simulation cell, and $N_{n b}$ is the number of neighbors of the ith particle. Previously, it was shown that this modified Lindemann parameter characterized the melting transition very well 38]. The melting in the $x$ and $y$ directions was characterized by the quantities $\left\langle u_{x}^{2}\right\rangle / d_{x}^{2}$ and $\left\langle u_{y}^{2}\right\rangle / d_{y}^{2}$, where the relevant distance in each direction, $d_{x}$ and $d_{y}$, are respectively, given by the mean distance between particles in each row, and the distance between chains. In the single-chain regime we study the behavior of $\left\langle u_{y}^{2}\right\rangle$ (not $L_{p y}$ ), since there is no characteristic distance in the $y$ direction. In the $x$ direction, the modified Lindemann parameter $\left\langle u_{x}^{2}\right\rangle / d_{x}^{2}$ can be defined normally, where $d_{x}$ is the average distance between particles along the chain.

Besides the modified Lindemann parameter described above, we also calculated other quantities in order to better characterize the melting phenomenon. An example is 
the correlation function $G_{\eta}$, given by:

$$
G_{\eta}=\frac{1}{N}\left\langle\sum_{i=1}^{N} \frac{1}{N_{n b}} \sum_{j=1}^{N_{n b}} \cos \left(\frac{2 \pi\left(\eta_{i}-\eta_{j}\right)}{d_{\eta}}\right)\right\rangle,
$$

where $\eta=x, y$ and $d_{\eta}=d_{x}, d_{y}$ (defined above). The function $G_{\eta}$ indicates how ordered a configuration is. Specifically, it compares the distance between particles for a given temperature with the distance between particles in the ground state configuration $(T=0)$. In the case of a highly ordered configuration, $G_{\eta}$ is equal to unity. For the completely disordered case the correlation function is zero. In the present study, the $G_{\eta}$ function is calculated for each type of particle separately. This means in the single- and two-chain configurations it is defined only in the $x$ direction.

\section{RESULTS}

In general, the present binary system can be characterized by an asymmetrical thermal behavior with respect to the spatial directions $x$ and $y$, and with respect to the two types of particles. Anisotropy with respect to the spatial direction was also found in the mono-disperse system [9], but we found here that the presence of different types of charges introduces new features. For some cases, a very restrictive thermal behavior is observed only for one type of particle.

As a representative example we study the case in which the ratio between charges is $\alpha=2$, and the screening parameter $\kappa=1$, which is a typical value for colloids and dusty plasmas [39]. The thermal stability of the structures will be analyzed for different densities. We will also pay special attention to the case when the distinct types of charges are almost equal $(\alpha \approx 1)$. Earlier we found that in this case, the particles are arranged in a mixed configuration where both types of charges are randomly distributed over the rows [10]. The zero temperature phase diagram of the present system can be found in Fig. 3 of Ref. [10].

We start by presenting the results for the one-chain regime, where the mean squared displacements have a very particular behavior as a function of temperature. Next, the multi-chain regime will be studied, where we concentrate on the two- and four-chain regimes. For the parameters $\alpha=2, \kappa=1$, and the interval of density considered here the system is found only in the minimum energy structures with one, two, and four chains [10]. Finally, the interesting mixed or disordered case is investigated.

\section{A. The single-chain regime}

The single-chain regime is very peculiar. In principle, a Lindemann-like criterion can not be applied here since for small densities the difference in the mean square

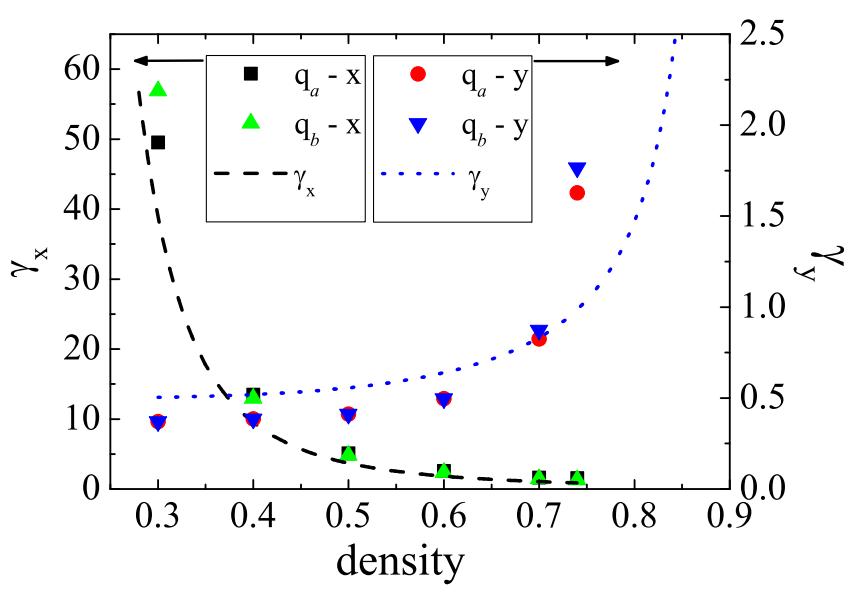

FIG. 2: (Color ) The slopes $\gamma_{x}$ and $\gamma_{y}$ in the low temperature interval of the curves $\left\langle u_{\eta}^{2}\right\rangle$ versus $T(\eta=x, y)$ obtained from simulations in the single-chain regime, and analytically as given by Eqs. (5) and (6) as a function of density.

displacement behaves linearly with temperature, and no clear melting transition is found. For high densities, the usual abrupt increase of the mean square displacement is found at some critical temperature which is characteristic for melting, but this is only found along the chain. In addition, the dimensionless modified Lindemann parameter presented in Sec. П is not defined in the $y$ direction, since there is no characteristic distance in this direction. In this case, we still study the thermal behavior of $\left\langle u_{y}^{2}\right\rangle$, without reference to a melting temperature.

For $\alpha=2$ and $\kappa=1$ the one-chain configuration [see Fig. 12 in Appendix A] is found in the interval of density $0<n \lesssim 0.74155$. For small densities, $n<0.5$, the mean square displacements $\left\langle u_{x}^{2}\right\rangle$ (and also $L_{p x}$ ) and $\left\langle u_{y}^{2}\right\rangle$ increase linearly with temperature, typical for harmonic oscillations of particles around their respective equilibrium positions [Fig. 1(a)]. The slopes of $\left\langle u_{x}^{2}\right\rangle$ and $\left\langle u_{y}^{2}\right\rangle$ are almost the same for the two distinct charges, but they are very different along the $x$ and $y$ directions, being a factor of almost 20 larger in the former. The reason for such an asymmetry can be qualitatively understood by the fact that a parabolic confinement in the $y$ direction is equivalent to a homogeneous distribution of background charge in that direction. This charge distribution is constant, independent of temperature, and generates a constant pressure in the direction perpendicular to the chain of charges. On the other hand, the pressure along the chain direction is a consequence of the interaction of each charge with all other particles in the chain. Such an interaction is strongly affected by the relative positions of the particles, i.e. the density, and increases with increasing density. For small densities the restoring force to bring the particles back to their equilibrium position is very small which explains the large values obtained for $\left\langle u_{x}^{2}\right\rangle$.

For $n \gtrsim 0.5$ the behavior of the quantity $\left\langle u_{y}^{2}\right\rangle$ remains 


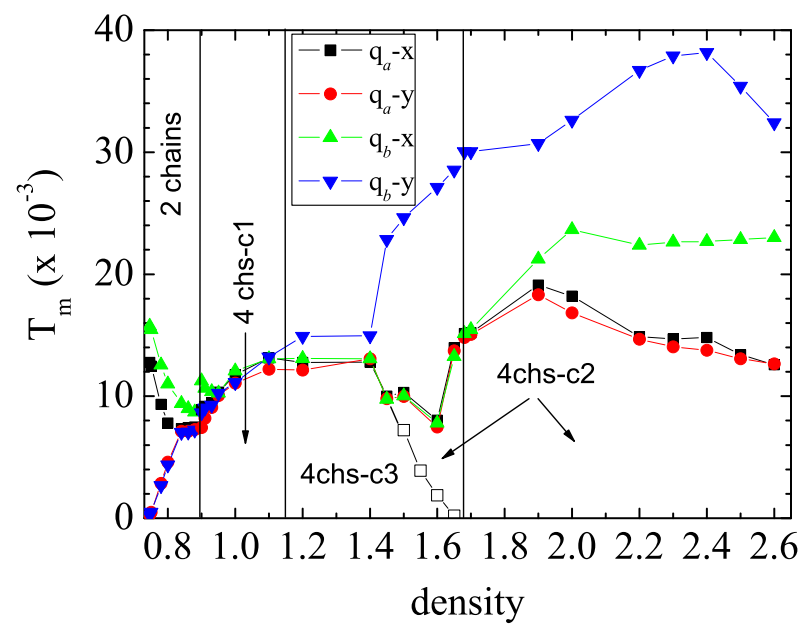

FIG. 3: (Color) The melting temperature of the distinct types of charges $\left(q_{a}=1, q_{b}=2\right)$ and different directions $(x$, $y$ ) for the multi-chain regime as a function of density $(n)$. The open-square symbols indicate the temperature for which a plateau in the $\left\langle u^{2}\right\rangle$ versus $T$ curves appear in the four-chain (case 3) regime.

linear with temperature, but the temperature dependence of $\left\langle u_{x}^{2}\right\rangle$ is qualitatively different. The harmonic oscillation (linear part) observed for small temperatures is now followed by a nonlinear regime, which becomes more abrupt for larger densities. As illustrated in Figs. 1(cd), the temperature at which $\left\langle u_{x}^{2}\right\rangle / d_{x}^{2}$ increases abruptly becomes smaller with increasing density, and depends on the type of particle. For larger densities, fluctuations of the particle positions can destabilize the ordered structure more easily. This behavior is very different from the melting of a classical 2D Wigner solid where the melting temperature has the dependence $T_{c} \sim \sqrt{n}$.

Another qualitative modification of the $\left\langle u^{2}\right\rangle$ versus $T$ curves with increasing density is related with the linear regime. Notice that for large densities the slope of the linear part of the $\left\langle u_{x}^{2}\right\rangle$ curves becomes smaller than the one in the $y$ direction. This is a consequence of the fact that in the $y$-direction there is an energy barrier for the zig-zag transition [9, 40], and the barrier height decreases with increasing density and consequently the effective potential minima become shallower.

An approximately analytic expression for the slope of the linear $T$-dependence of $\left\langle u_{\eta}^{2}\right\rangle$ can be obtained . Consider the potential energy given by Eq. (2), next we limit ourselves to the nearest neighbors and then expand the Yukawa interaction terms with respect to small oscillations. In this way we obtain the deviation of the particles, within the harmonic approximation, from which we obtain expressions for $\left\langle u_{\eta}^{2}\right\rangle$. Within the harmonic approximation we have $\left\langle u_{\eta}^{2}\right\rangle=\frac{1}{2} k_{B} T$ from which we found, in dimensionless units, the slopes

$$
\gamma_{x} \approx\left[4 \alpha n^{2} \kappa \exp (-\kappa / n)\right]^{-1},
$$

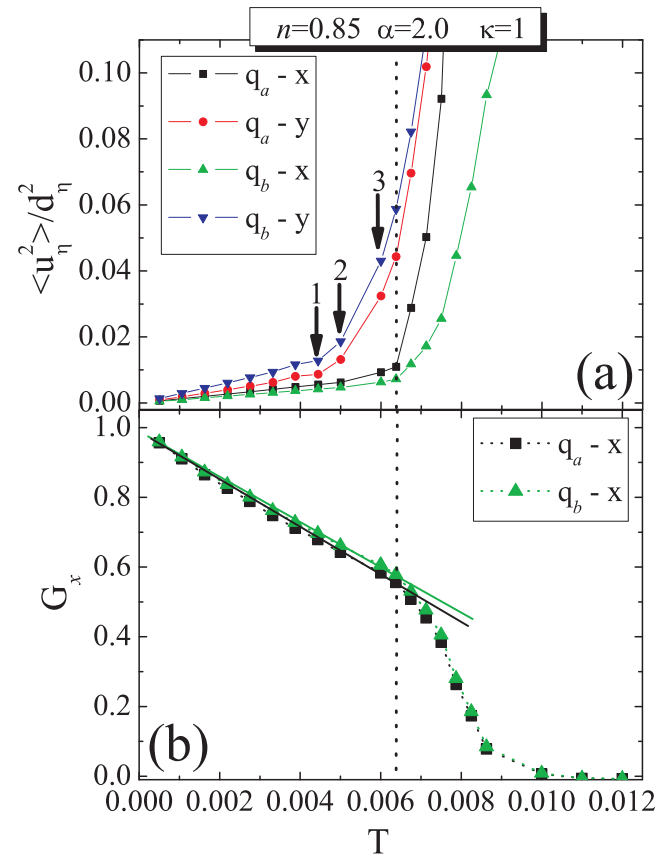

FIG. 4: (Color ) (a) The Lindemann parameter $\left\langle u_{\eta}^{2}\right\rangle / d_{\eta}^{2}(\eta=$ $x, y)$ for charges $q_{a}=1$ and $q_{b}=2$ ), and (b) the correlation function $G_{x}$ as a function of temperature for a system with density $n=0.85, \alpha=2$ and $\kappa=1$ (two-chain configuration). The vertical dotted lines cross the full lines at $G_{x}=0.5$.

and

$$
\gamma_{y} \approx\left[2-2 \alpha n^{2}(n+\kappa) \exp (-\kappa / n)\right]^{-1},
$$

where the density in the single-chain regime is $n=1 / a$, with $a$ the distance between neighbor charges along the chain. From Eqs. (5) and (6) we note that the slope $\gamma_{y}$ increases with density. In the $x$ direction, the opposite behavior is found for $\gamma_{x}$. These approximate analytic expressions are compared with the results from our simulations in Fig. 2. In spite of the rough approximation used to obtain Eqs. (5) and (6), there is good qualitative agreement with the numerical results, especially in the $x$ direction, which corroborates with the qualitative explanation commented before. Note that from Eq. (6) we find that $\gamma_{y}$ diverges for $n \approx 0.897$ which signals the zigzag transition and compares with the value $n=0.74155$ which we found previously from our MC calculation. The difference is due to non-linear effects and contributions of particles beyond the nearest neighbor.

\section{B. The multi-chain regime}

In this subsection the thermal behavior for the twoand four-chain structures are presented (Note that a three-chain structure is not stable; see Fig. 12 in Appendix A). According to Ref. [10], the latter phase can be found in two different configurations, defined as case 
1 and case 2. However, we find here that a new fourchain (case 3) configuration is possible between these two phases. The new phase diagram is shown as Fig. 12 of Appendix A and replaces Fig. 3 of Ref [10].

A general feature of the multi-chain regimes is an asymmetrical melting in both the direction and the distinct types of particles. In the two- and four-chain (case 2) regimes particles with smaller charge melt first. This is not the case for the four-chain (case 1) system.

\section{The two-chain regime}

For $n=0.74155$ the system undergoes a continuous structural phase transition from the one- to the two-chain configuration [10]. For the two-chain configuration, the melting is anisotropic with respect to the charges and with respect to the spatial directions $x$ and $y$. The corresponding melting temperatures are summarized in Fig. 3. As can be seen in Fig. 3 for both types of particles, the melting temperature increases with increasing density in the $y$ direction, while the opposite trend is found in the unconfined $x$ direction. The explanation for such a different dependence is associated with the fact that the repulsive Coulomb interaction increases when the density increases. Such an increase in the Coulomb repulsion leads to a dramatic different behavior in the $x$ and $y$ directions. In the latter, and in association with the external confinement potential, the amplitude of the oscillations are smaller, which explains the behavior of the melting temperature in the $y$ direction [Fig. 3 . The equilibrium along the $x$ direction is established by the balance of the electrostatic force between charges. Thermal fluctuations of the particle positions destroy such balance and, consequently, the ordered arrangement. A larger density brings the charges closer to one another, increasing the repulsive interaction. In this case, small fluctuations of the particle positions (small temperature) are enough to destabilize the ordered structure, and to melt the system in the unconfined direction. This different behavior along the $x$ and $y$ directions is in line with the results found for a single chain as presented in Fig. 2 .

In Fig. 3 we see that for a given density, we find in the two-chain regime a smaller melting temperature in the $y$ direction. Along the unconfined direction, the melting temperature is different for both types of particles, being larger for the bigger charge. This result is obtained according to the modified Lindemann criterion $\left(\left\langle u_{\eta}^{2}\right\rangle / d_{\eta}^{2}=0.1\right)$, as illustrated in Fig. 4 (a) for a system with density $n=0.85$. This conclusion is corroborated by our study through the correlation function $G_{x}$ defined by Eq. (4). As shown in Fig. 4(b), similar qualitative and quantitative behavior is found for both charges along the unconfined direction, where the $G_{x}$ value for the $a$ particles is slightly smaller which is indicative for a lower melting temperature.

In Fig. 5, we present the distribution of particles along

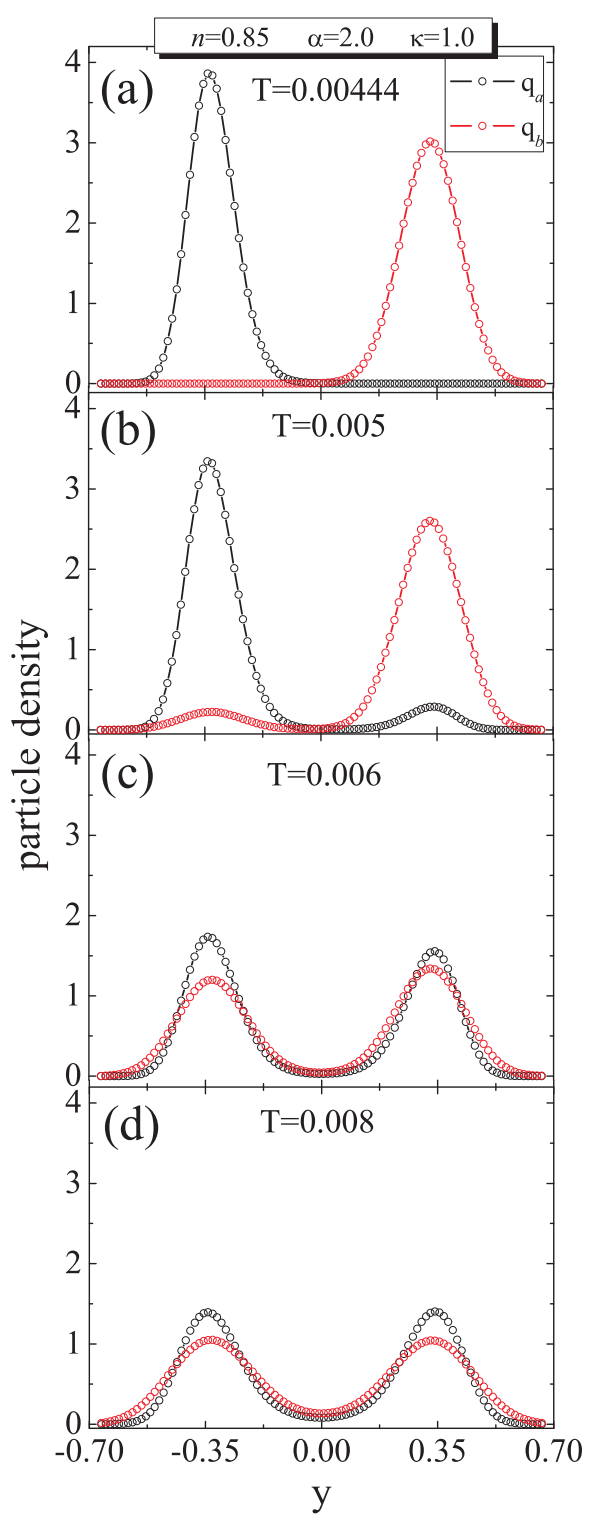

FIG. 5: (Color ) The distribution of particles along the confined direction in the two-chain regime $(n=0.85, \alpha=2$ and $\kappa=1$ ), for different temperatures (a) $T=0.00444$, (b) $T=0.005$, (c) $T=0.006$, and (d) $T=0.008$.

the $y$ direction for the same system (density $n=0.85$ ), and for different temperatures. Three of those temperatures, namely $T=0.00444, T=0.005$, and $T=0.006$, are indicated in Fig. 4(a) by arrows 1, 2, 3, respectively. For all temperatures the distribution of particles is asymmetric along the $y$ direction, and particles with larger charge are more spread out along the confined direction, which is due to the larger Coulomb repulsion. For small temperature (e.g., $T=0.00444$ ) the distinct types of particles are segregated in different chains [Fig. [5(a)], while with increasing temperature the two types of particles become more and more mixed over both chains [Fig. 5(d)]. 


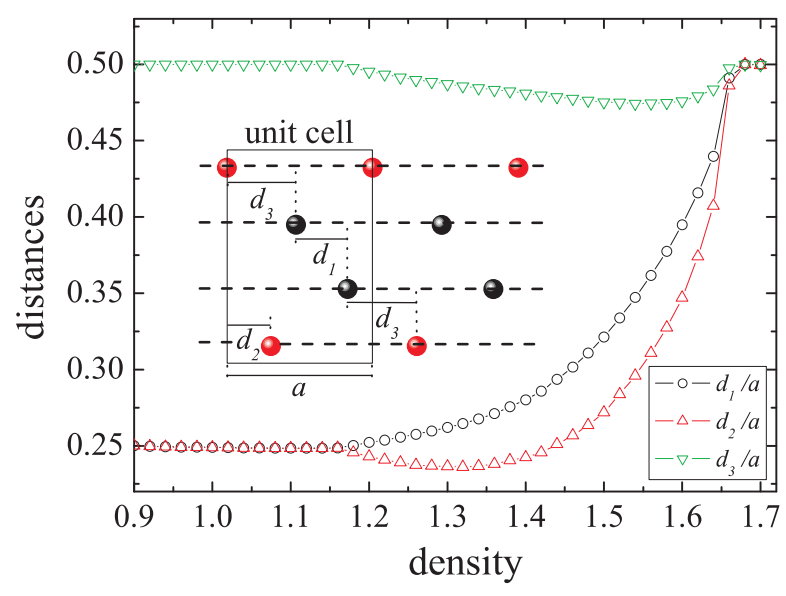

FIG. 6: (Color) The distances $d_{1} / a, d_{2} / a, d_{3} / a$, which characterize the four-chain (case 3 ) regime, as a function of density for a system with $\kappa=1$ and $\alpha=2$. A sketch of the ground state configuration is presented as inset. $a$ is the mean distance between particles along each chain.

The results shown in Fig. 5 indicate that the melting in the $y$ direction is not an abrupt process. For $T=0.005$ [arrow 2 in Fig. 4(a)], particles already start to jump between chains, which does not occur in the linear regime of $\left\langle u_{y}^{2}\right\rangle / d_{y}^{2}$ [arrow 1 in Fig. 4(a)]. Note that for $T=0.006$ [arrow 3 in Fig. 4(a)], which is smaller than the melting temperature given by the Lindemann-like criterion, particles of both chains are already mixed, suggesting that the melting process in the $y$ direction is not abrupt, and starts with a non-linear increase of the mean square

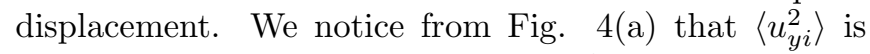
a rather continuous function while $\left\langle u_{x i}^{2}\right\rangle$ exhibits a very steep increase at a certain temperature.

Along the chain direction, the temperature for which a deviation from the linear decay of $G_{x}$ occurs [Fig. 4(b)], coincides with the one along the $x$ direction for which the Lindemann parameter deviates from a linear temperature behavior [Fig. 4(a)]. Note that in this case, the deviation of the Lindemann parameter from the linear regime starts at the same temperature $(T=0.007)$ for both types of charges [Fig. 4(a)] as observed in Fig. 4(b) for $G_{x}$. In the unconfined direction, the correlation function together with $\left\langle u_{x i}^{2}\right\rangle$ suggests a continuous disordering of the system with increasing temperature.

\section{The four-chain (case 1 and case 3) regimes}

A further increase in the density brings the system to the four-chain regime, which is found in three different configurations. In all of them (case 1, case 2, case 3 ), the different types of particles are symmetrically distributed with respect to the $x$-axis, but segregated in distinct chains. In this section we discuss the thermal behavior of case 1 and case 3 .
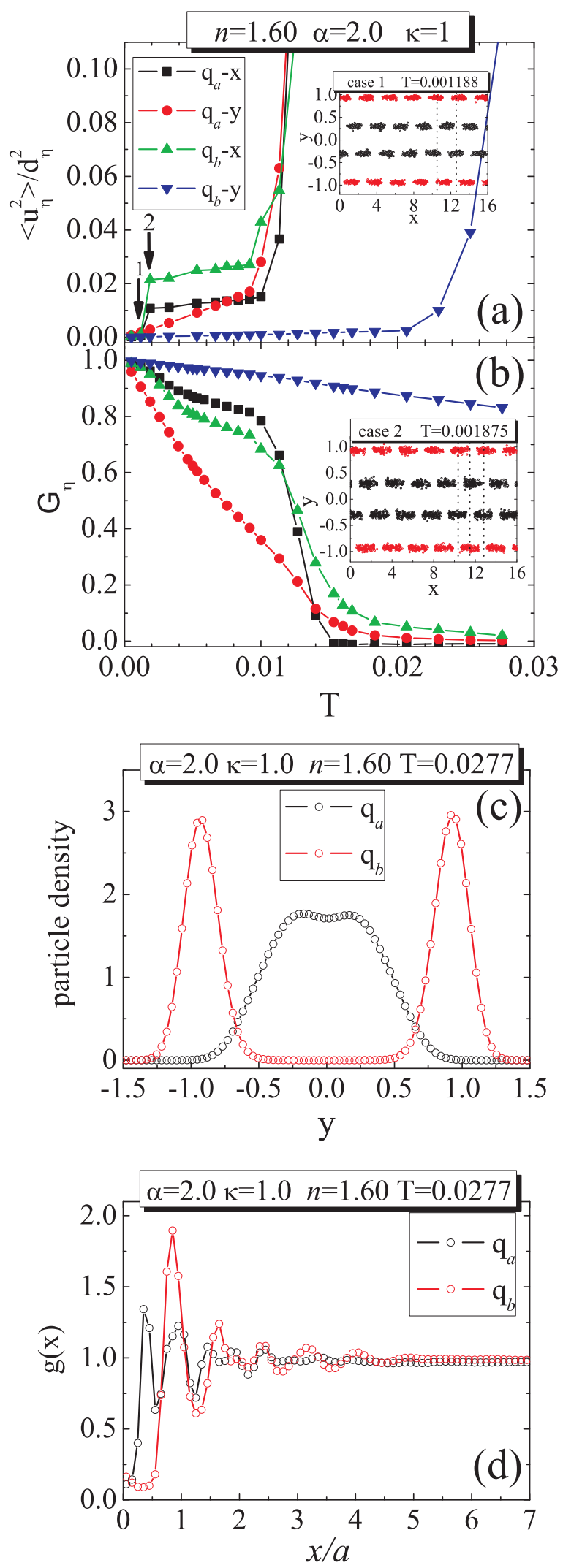

FIG. 7: (Color) (a) The Lindemann parameter $\left\langle u_{\eta}^{2}\right\rangle / d_{\eta}^{2}$, and (b) the correlation function $G_{\eta}(\eta=x, y)$ for charges $q_{a}=1$ and $q_{b}=2$ as a function of temperature for a system with density $n=1.60, \alpha=2$ and $\kappa=1$. Particle trajectories over $100 \mathrm{MC}$ steps for temperatures $T=0.001188$ (arrow 1) and $T=0.001875$ (arrow 2) are shown as insets in (a) and (b), respectively. Black (red) circles represent particles with charge $q_{a}=1\left(q_{b}=2\right)$. (c) The distribution of particles in the $y$ direction. (d) The pair-correlation function along the $x$ direction. $a$ is the mean distance between particles along 
In the four-chain (case 1) regime, which is found in the density interval $0.9 \lesssim n \lesssim 1.16$, chains with equal charges are displaced with respect to each other by $a / 4$ ( $a$ is the distance between particles in each chain) along the chain direction ( $x$-axis), while neighbor rows with distinct types of particles are displaced by a distance $a / 2$ along the $x$-axis. The distance between the internal chains (consisting of particles with the same charge) is larger than the distance between the internal chains and the external ones [10]. This is interesting because the interaction between chains with distinct charges is intuitively expected to be larger than the interaction between chains with the same lower charge. In the case 1 regime the melting temperature in both spatial directions and for the two different particles is almost the same as shown in Fig. 3 ,

For $1.16 \lesssim n \lesssim 1.68$ the system suffers a continuous transition to the four-chain (case 3) regime which was not predicted in Ref. [10]. Details of the internal configuration of particles in such a regime is presented as inset in Fig. 6. The main difference with respect to the case 1 regime is the density dependence of the distances $\left(d_{1}, d_{2}, d_{3}\right)$ which characterizes the ground state configuration. In the four-chain (case 1 ) regime $d_{1}=d_{2}=a / 4$, and $d_{3}=a / 2$ ( $a$ is the distance between particles along each chain). As will be presented in the next section, the four-chain (case 2) configuration is characterized by $d_{1}=d_{2}=d_{3}=a / 2$.

The behavior of the melting temperature in the fourchain (case 3) regime is rather distinct when compared to the one in case 1 . There is a sudden increase of the melting temperature of the external chains in the $y$ direction for $n>1.4$. On the other hand, the melting temperature for the internal chains decreases with increasing density. The same decrease in the melting temperature is observed for the external chains in the $x$ direction. The reason for the modified density dependence of the melting temperature is a thermally structural phase transition in which the system changes from the four-chain (case 3) to the four-chain (case 2) configuration. For example, in Fig. 7(a) the Lindemann parameter $\left\langle u_{\eta}^{2}\right\rangle / d_{\eta}^{2}(\eta=x, y)$ for charges $q_{a}$ and $q_{b}$ is presented as function of temperature for a system with $n=1.60$. As can be seen, there is a small plateau in the mean square displacement for both charges, but only in the unconfined direction. As observed previously in the case of circularly confined finite size clusters [34], the plateau is a typical signature of a thermally induced structural phase transition, and we find here that the plateau is, in fact, associated with such a transition. In the insets in Figs. 7(a) and 7(b), we present typical trajectories of the particles obtained over $100 \mathrm{MC}$ simulation steps for temperatures below and above the plateau [indicated by the arrows 1 and 2 in Fig. 7(a)]. Around the structural phase transition the system is in the harmonic regime, since the mean square displacement increases linearly with temperature. For temperatures before the plateau, the particles are arranged in the four-chain (case 3) regime, while above the plateau the trajectories of the particles indicate that the system is in the four-chain (case 2) regime [10], whose features are described in the next section. Such a thermally induced structural phase transition is observed only for densities $n \gtrsim 1.50$ in the four-chain (case 3 ) regime. The temperature for which the plateaus appears as a function of density is indicated in Fig. 3 by the open square symbols. Above those temperatures the system is found in the case 2 regime, as indicated in Fig. 3 ,

From the analysis of the Lindemann parameter, we find that the thermal behavior depends on the spatial direction, which is qualitatively similar for both types of particles. However, if the correlation function $G_{\eta}(\eta=x, y)$ defined in Eq. (4) is considered [Fig. 7(b)], we still observe a similar thermal dependence in the $x$ direction for the charges $q_{a}$ and $q_{b}$. But this is different in the $y$ direction. The thermal dependence of $G_{y}$ for charge $q_{a}$ (internal chains) suggests a continuous loss of ordering with increasing temperature, and differs from the one of particles with charge $q_{b}$ (external chains). The latter thus indicates an ordered structure due to the balance between the Coulomb repulsion from the charges in the internal chains and the external pressure from the parabolic confinement potential.

For high temperatures, e.g. $T=0.0277$, the internal chain-like structure of the system is disappeared, while the external chains still remain well defined [Fig 7(c)]. However, for both internal and external chains a liquid-like behavior is found along the chain direction. The mean distance between particles with smaller charge $\left(q_{a}\right)$ in the $x$ direction becomes half the distance between particles with larger charge $\left(q_{b}\right)$. This is also illustrated by Fig. 7(d), where the pair correlation function $g(x)=\frac{L}{N^{2}} \sum_{i \neq j}\left\langle\delta\left[x-\left(x_{i}-x_{j}\right)\right]\right\rangle$ is shown.

\section{The four-chain (case 2) regime}

For $T=0$ there is a continuous structural phase transition, at $n \approx 1.68$, from case 3 to case 2 (see Fig. 6). In the four-chain (case 2) regime, chains with the same charge are displaced by $a / 2$ with respect to each other along the $x$ direction. The density dependence of the distance between chains has an opposite behavior to the one of case 1, i.e. the distance between chains with distinct charges is larger than the distance between the internal rows [10]. The different internal structure of the fourchain (case 2) regime leads to a different temperature dependence for the mean square displacements. As can be observed in Fig. 3, the melting temperature for both types of particles, and in the two directions has a different behavior as a function of density in the two phases of the four-chain regime.

The particular arrangement of the charges [see inset in Fig. 6] results in a larger distance between the internal $\left(q_{a}\right)$ and the external $\left(q_{b}\right)$ chains than that found in case 1. As a consequence, the external confinement potential acts more strongly on the particles placed in the outer 

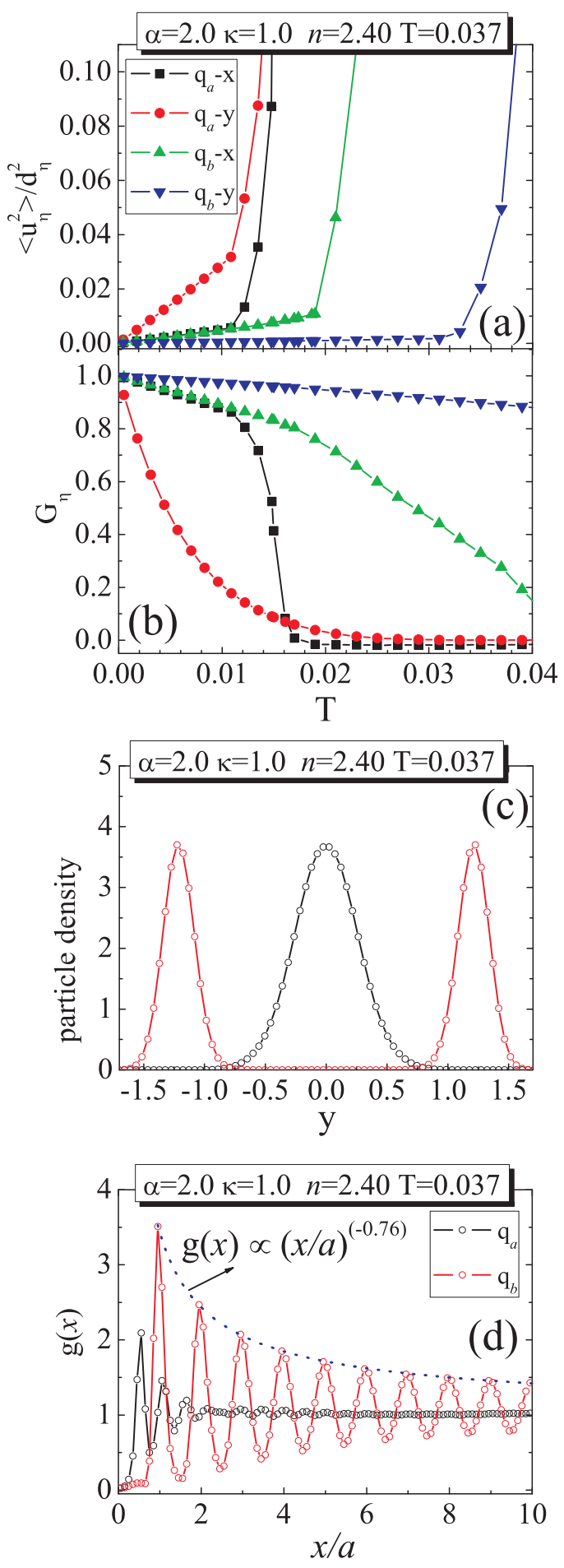

FIG. 8: (Color) (a) The Lindemann parameter $\left\langle u_{\eta}^{2}\right\rangle / d_{\eta}^{2}$, and (b) the correlation function $G_{\eta}(\eta=x, y)$ for charges $q_{a}$ and $q_{b}$ as a function of temperature for a system with density $n=2.40, \alpha=2$ and $\kappa=1$. (c) The distribution of particles in the $y$ direction. (d) The pair-correlation function along the $x$ direction. $a$ is the mean distance between particles along each chain in the ground state configuration. The peaks of the pair correlation function for the $b$-particles is fitted by an algebraic decay represented by the dotted blue curve. The legend in (b) is the same of (a), and the legend in (d) is the same of (c). rows, resulting in a larger melting temperature in the $y$ direction. Fig. 3 indicates a clear anisotropy in the melting temperature of the external chains with respect to the $x$ and $y$ directions.

Now, considering the inner chains we observe that particles in different rows are already close such that fluctuations of the particle positions are sufficient to destroy the ordering in both directions. This fact occurs for a temperature smaller than those needed to melt the external chains. We can conclude that according to the modified Lindemann criterion considered in the present paper, the melting in the four-chain (case 2) regime is anisotropic with respect to both spatial directions and the distinct types of charges.

We resort again to the correlation function $G_{\eta}(\eta=$ $x, y)$ and other quantities in order to better understand the temperature dependence. As an example, in Figs. 8 (a) and 8 (b), a comparison between the temperature dependence of the Lindemann parameter and the correlation function $G_{\eta}$ for density $n=2.40$ is made. In both the $x$ and $y$ directions the Lindemann parameter has the same qualitative behavior as a function of temperature for both types of particles, i.e. a linear behavior followed by an abrupt non-linear increase of $\left\langle u_{\eta}^{2}\right\rangle / d_{\eta}^{2}$ [Fig. 8(a)]. On the other hand, $G_{\eta}$ presents a very different temperature dependence in the $x$ and $y$ direction, and for both types of charges. As in the case 1 regime, $G_{y}$ shows a continuous loss of ordering as a function of $T$ for the internal chains $\left(q_{a}\right)$, which is not observed for the external ones. In the $x$ direction, the behavior of the internal and external chains is similar only for small temperatures $(T \lesssim 0.01)$. For $T \gtrsim 0.01$, the behavior of $G_{x}$ for the internal chains exhibits an abrupt decay in the corresponding temperature interval associated to the melting according to the Lindemann parameter criterion. For particles in the external chains, we find a $G_{y}$ $T$-dependence that suggests a slowly disordering with increasing temperature, which is not seen from the Lindemann parameter [Fig. 8(a)]. For example, at the temperature $T=0.037$ the Lindemann parameter indicates that the system is completely melted, but $G_{y}$ indicates that only the internal chains are completely disordered (i.e., $G_{x} \approx G_{y} \approx 0$ ).

In Figs. $8(\mathrm{c})$ and $8(\mathrm{~d})$, we show the distribution of particles along the $y$ direction and the pair correlation function along the unconfined direction for $T=0.037$, respectively. As can be observed, the initial two-chain structure of the internal chains is completely destroyed in the $y$ direction, while it is relatively preserved in the external chains. Along the $x$ direction, Fig. 8(d) indicates that the internal chains are completely disordered, but the pair-correlation function for particles in the external chains presents an algebraic decay, which is indicative of long-range order. Notice that the $G_{x}$ function for the $b$ particles still presents a finite value for $T=0.037$ in agreement with the $g(x)$ result. For very high temperatures, and as found in the case 3 regime, the mean distance between the particles ( $x$ direction) with smaller 


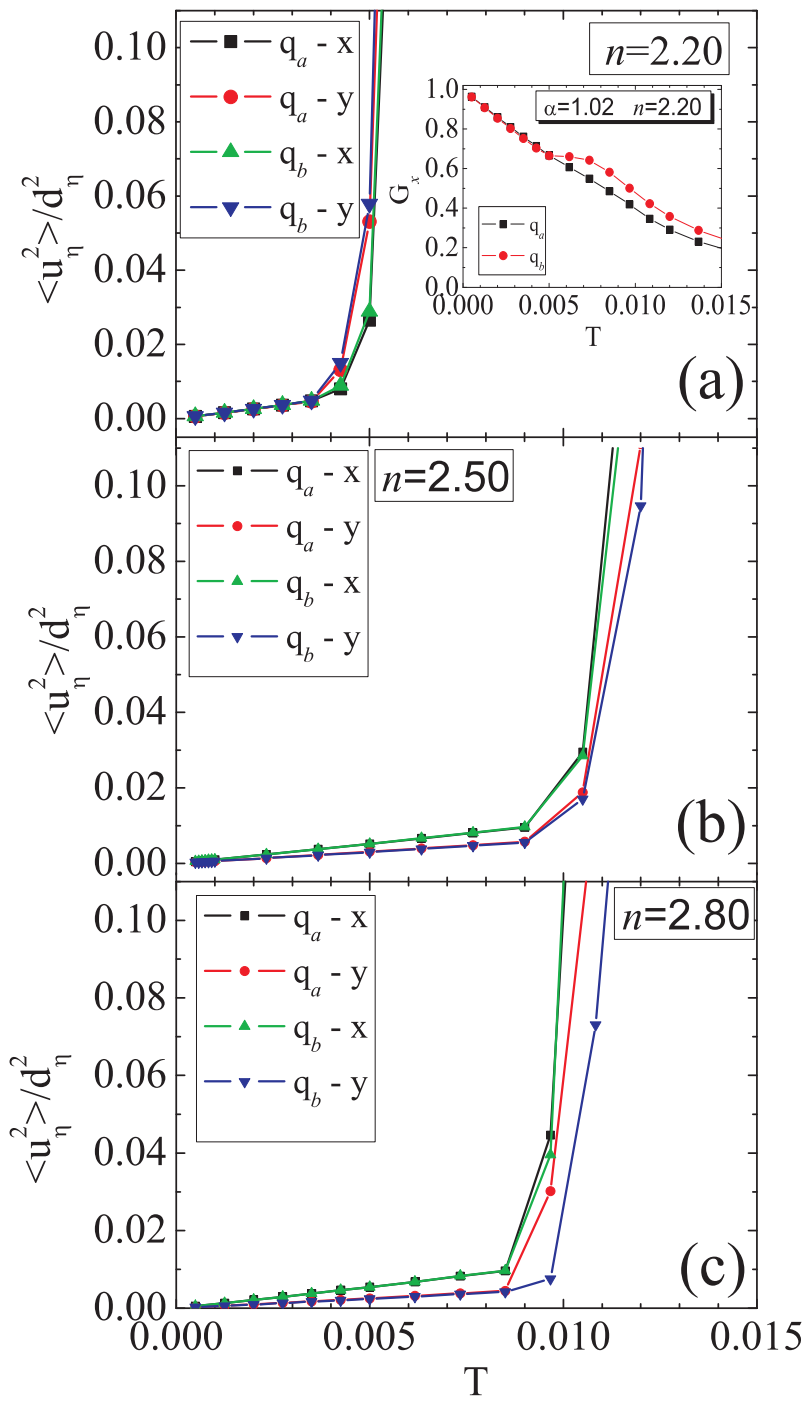

FIG. 9: (Color) The Lindemann parameter $\left\langle u_{\eta}^{2}\right\rangle / d_{\eta}^{2}$ for a system in the mixed regime $\left(q_{a}=1\right.$ and $\left.q_{b}=1.02\right)$ as a function of temperature for different densities (a) $n=2.20$, (b) $n=2.50$, and (c) $n=2.80$.

charge becomes half the one for larger charge (first peak in the pair correlation function), which indicates that a disordered one chain is found in the internal part of the system. The results presented here indicate a complete anisotropic (direction and type of charge) melting for the binary system.

\section{Mixed regime}

In the almost mono-disperse case (i.e. $\alpha \approx 1$ ), the minimum energy arrangement is the three-chain configuration with the charges randomly distributed over the chains. Neighbor chains are displaced by a distance $a / 2$ with respect to each other along the unconfined direction

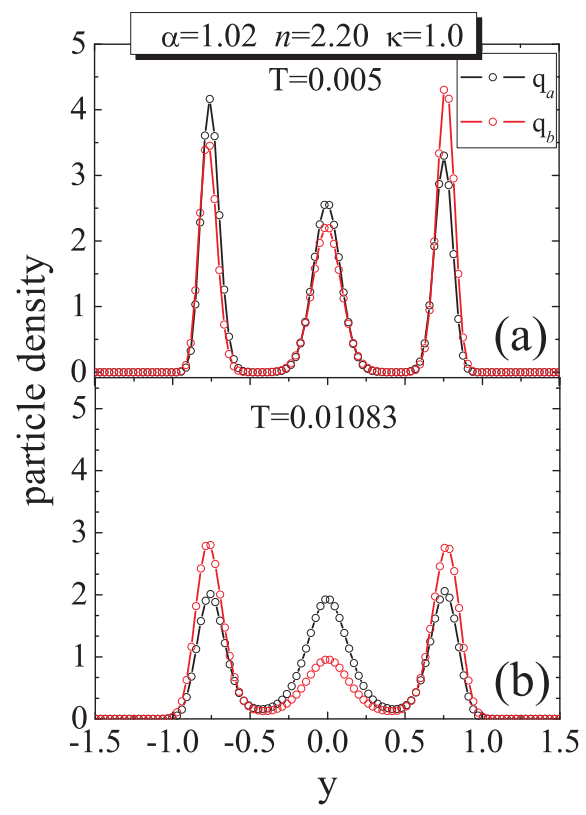

FIG. 10: (Color) The distribution of particles $\left(q_{a}=1\right.$ and $q_{b}=1.02$ ) along the confined $y$ direction for a system in the mixed regime for temperatures (a) $T=0.005$, and (b) $T=0.01083$.

[inset of Fig. 11]]. For $\kappa=1$, the interval of density in which the mixed regime can be observed depends on the ratio between the size of the charges $\alpha$ [10]. Here, we focus on the representative case $\alpha=1.02$, i.e. particles have a difference in their charges of $2 \%$ where, for $T=0$, the disordered state was found in the density interval $2.14 \lesssim n \lesssim 3.23$.

The temperature dependence of the Lindemann parameter is presented in Fig. 9 for systems with $\alpha=1.02$, $\kappa=1$, and densities $n=2.20,2.50,2.80$. In spite of the small density interval in which the mixed regime is found [10], a different qualitative behavior is observed with increasing density. In general, the melting temperature increases with increasing density. In addition, the melting temperature in the $y$ direction becomes distinct for both types of particles. In the $x$ direction, the melting temperature depends on the density, but it is the same for the two set of particles.

The random arrange in the mixed regime generates an unbalanced distribution of the different charges over the chains, as can be seen in Fig. 10(a), where the distribution of particles along the confined direction is shown for the system with density $n=2.20(\alpha=1.02, \kappa=1)$ at $T=0.005$. An interesting feature is observed when the temperature is increased. In this case, a more homogeneous distribution of both types of particles is induced, where particles with larger charge are located predominantly at the edge chains [Fig. [10(b)]. A similar behavior is found for all densities in the mixed regime. It is interesting to comment on the more homogeneous distribution 


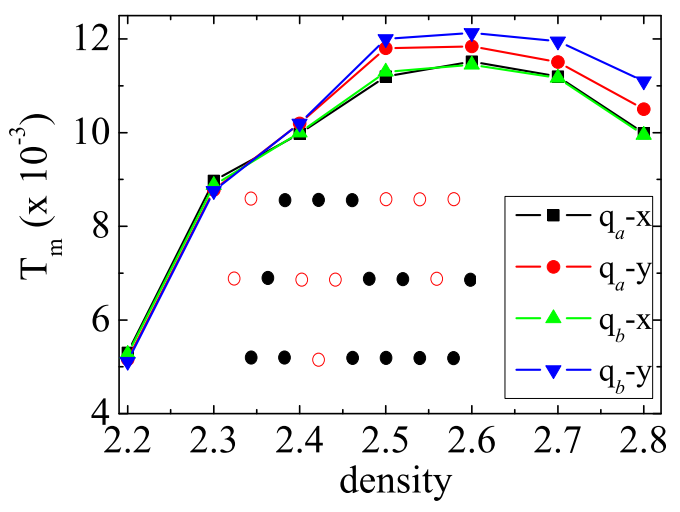

FIG. 11: (Color) The melting temperature of the distinct types of particles $\left(q_{a}=1\right.$ and $\left.q_{b}=1.02\right)$ as a function of density for a system in the mixed regime. An example of the ground state configuration is shown as inset

of particles which can also be noticed from the correlation function. In particular, we present as inset in Fig. 9(a) the correlation function $G_{x}$ as a function of temperature for the system with $n=2.20(\alpha=1.02, \kappa=1)$. For $T \gtrsim 0.005$ the correlation function $G_{x}$ is larger for particles with larger charge $\left(q_{b}=1.02\right)$, which coincides with the rapid increase of $\left\langle u_{y}^{2}\right\rangle$ at which particles start to jump between chains leading to a more homogeneous distribution of the different particles over the chains. At this point the system melts. The density dependence of the different melting temperatures of the system with $\alpha=1.02$ and $\kappa=1$ in the mixed regime is summarized in Fig. 11,

\section{CONCLUSION}

We studied melting and thermal induced transitions of a two-dimensional binary system consisting of an equal number of particles with different charges interacting through a screened Coulomb potential, and confined along one direction by a parabolic potential. The different phases (see Fig. 12) that nucleate as a function of density [10] exhibit a very distinct and nontrivial temperature dependence.

The melting temperature was determined from a modified Lindemann criterion [9]. Other quantities as the pair-correlation function, the distribution of particles, and the translational correlation function were in addition studied to corroborate the obtained values for the melting temperature and to enhance our understanding of the melting process of the different ordered structures.

We found that melting is anisotropic with respect to the spatial $x$ and $y$ directions, and with respect to the distinct type of particles. In the multi-chain regime, depending on the internal structure of the system, a very different temperature behavior is found. Specifically, in the new four-chain (case 3) regime we found a thermally induced structural phase transition, which shows up by the appearance of a plateau in the curves of the mean square displacement versus temperature. For the fourchain (case 2) regime an analysis of the pair-correlation function shows that the external chains, composed by particles with larger charge, exhibits a relatively ordered structure along the unconfined direction, even for a temperature larger than the melting temperature as determined by the Lindemann-like criterion. The correlation function, indicates a qualitatively distinct melting in the $x$ and $y$ directions and for the distinct types of particles.

To summarize, the two- to four-chain transition was found previously to be a first order transition $($ at $T=0)$ ) and we found here that the density at which the transition occurs is independent of temperature. The different melting temperatures exhibit small jumps at this phase transition. This is different for the different four-chain regimes, which was previously found to crystallize only in two configurations with a first order transition between them. Here we predict that a new four-chain phase exist intermediate between the previous two four-chain configurations that we studied in Ref. [10]. As a consequence, the transitions between the different four-chain phases are now continuous, i.e. second order, instead of first order transitions. In addition, the density at which the transition takes place depends on temperature which is a qualitative difference from the mono-disperse case [9].

For $\alpha \approx 1$, the system can be found in a mixed configuration which we studied in the regime of three chains. The configuration is characterized by a random distribution of the two types of charges over the chains. In this regime, we found that an increase of temperature results in a more symmetric distribution of particles over the different chains, and an increase of the melting temperature with density, except for high densities.

The predicted melting phase diagram for the binary quasi-one-dimensional system can be investigated experimentally in systems as e.g. dusty plasmas and colloids. Previously, harmonic confinement potentials have been realized in dusty plasma [22] and in colloidal 37] systems. The phase diagram can be scanned experimentally by changing e.g. the density. Furthermore, in a dusty plasma it is also possible to vary the effective temperature which is not possible for a colloidal system where the particles move in a liquid environment. But for the latter system one can also use paramagnetic particles which, in the presence of a perpendicular magnetic field, become magnetized resulting in a dipole inter-particle interaction potential. The strength of this potential can be tuned by the external magnetic field [25]. Because the obtained results are generic, and qualitatively do not depend on the specific functional form of the repulsive interaction potential, similar melting behaviors are predicted for such a system of dipoles. Because in classical melting it is the thermal energy versus the average interaction energy that is the relevant parameter the temperature scale in Fig. 3 can also be replaced by the inverse of the interaction energy. 


\section{ACKNOWLEDGMENTS}

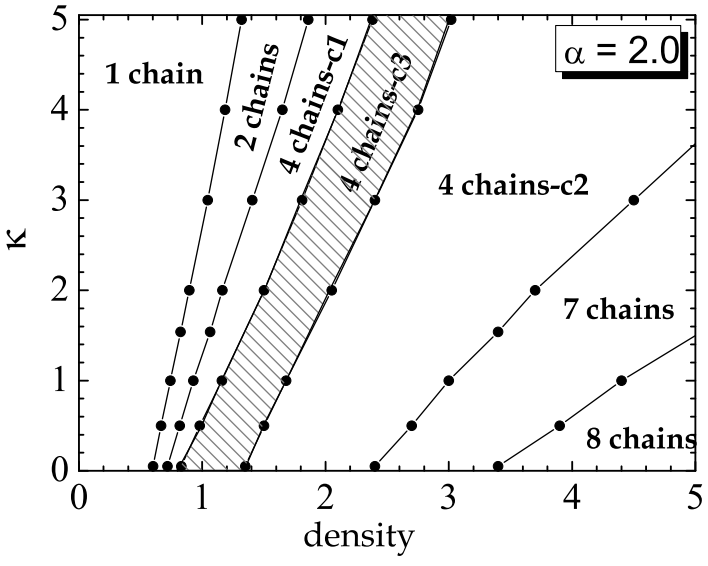

FIG. 12: (Color) The zero-temperature $\kappa$-density phase diagram for $\alpha=2$. The new stability region for the four-chain configuration is hatched.
This work was supported by CNPq, the Flemish Science Foundation (FWO-Vl) and the bilateral program between Flanders and Brazil.
[1] M. E. Leunissen, C. G. Christova, A. P. Hynninen, C. P. Royall, A. I. Campbell, A. Imhof, M. Dijkstra, R. van Roji, A. van Blaaderen, Nature (London) 437, 235 (2005).

[2] Y. H. Liu, Z. Y. Chen, M. Y. Yu, and A. Bogaerts, Phys. Rev. E 74, 056401 (2006); ibid, Phys. Rev. E 73, 047402 (2006).

[3] E. V. Shevchenko, D. V. Talapin, N. A. Kotov, S. O'Brien, and C. B. Murray, Nature (London) 439, 55 (2006).

[4] George K. Kaufman, Meital Reches, Samuel W. Thomas III, Ji Feng, BryanF. Shaw, and George M. Whitesides, Appl. Phys. Lett. 94, 044102 (2009).

[5] S. D. Overduin and C. N. Likos, Europhys. Lett. 85, 26003 (2009).

[6] Lahcen Assoud, René Messina, and Harmut Löwen, J. Chem. Phys. 129, 164511 (2008).

[7] W. P. Ferreira, F. F. Munarin, K. Nelissen, R. N. Costa Filho, F. M. Peeters, and G. A. Farias, Phys. Rev. E 72, 021406 (2005).

[8] W. P Ferreira, F. F. Munarin, F. M. Peeters, and G. A. Farias, J. Phys.: Condens. Matter 18, 9385 (2006).

[9] G. Piacente, I. V. Schweigert, J. J. Betouras, and F. M. Peeters, Phys. Rev. B 69, 045324 (2004).

[10] W. P. Ferreira, J. C. N. Carvalho, P. W. S. Oliveira, G. A. Farias, and F. M. Peeters, Phys. Rev. B 77, 014112 (2008).

[11] B. G. Levi, Phys. Today 41,(issue 9) 17 (1988).

[12] M. Drewsen, C. Brodersen, L. Hornekær, J. S. Hangst, and J. P. Schiffer, Phys. Rev. Lett. 81, 2878 (1998).

[13] B. A. Grzybowski, X. Jiang, H. A. Stone, and G. M. Whitesides, Phys. Rev. E 64, 011603(2001).

[14] S. G. Psakhie, K. P. Zolnikov, L. F. Skorentsev, D. S. Kryzhevich, and A. V. Abdrashitov, Phys. Plasmas 15, 053701 (2008).
[15] N. Hoffmann, F. Ebert, C. N. Likos, H. Löwen, and G. Maret, Phys. Rev. Lett. 97, 078301 (2006).

[16] E. P. Wigner, Phys. Rev. 46, 1002 (1934).

[17] C. C. Grimes and G. Adams, Phys. Rev. Lett. 42, 795 (1979).

[18] Oliver Arp, Dietmar Block, Andr Melzer, and Alexander Piel, Phys. Rev. Lett. 93, 165004 (2004).

[19] K. Zahn, R. Lenke, and G. Maret, Phys. Rev. Lett. 82, 2721 (1999).

[20] M. Golosovsky, Y. Saado, and D. Davidov, Phys. Rev. E 65, 061405 (2002).

[21] J. H. Chu and Lin I, Phys. Rev. Lett. 72, 4009 (1994).

[22] Bin Liu, K. Avinash, and J. Goree, Phys. Rev. Lett. 91, 255003 (2003).

[23] Bin Liu and J. Goree, Phys. Rev. E 71, 046410 (2005).

[24] Ramin Haghgooie, Chen Li, and Patrick S. Doyle, Langmuir 22, 3601 (2006).

[25] M. Köppl, P. Henseler, A. Erbe, P. Nielaba, and P. Leiderer, Phys. Rev. Lett. 97, 208302 (2006).

[26] Ramin Haghgooie and Patrick S. Doyle, Phys. Rev. E 70, 061408 (2004); Phys. Rev. E 72, 011405 (2005).

[27] Patrick S. Doyle, Jérôme Bibette, Aurélien Bancaud, and Jean-Louis Viovy, Science 295, 2237 (2002).

[28] Wen Yang, Kwinten Nelissen, Minghui Kong, Zhi Zeng, and F. M. Peeters, Phys. Rev. E 79, 041406 (2009).

[29] J. M. Kosterlitz and D. J. Thouless, J. Phys. C 61181 (1973).

[30] B. I. Halperin and D. R. Nelson, Phys. Rev. Lett 41, 121 (1978).

[31] A. P. Young, Phys. Rev. B 19, 1855 (1979).

[32] P. S. Branício, J. P. Rino, and N. Studart, Phys. Rev. B 64, 193413 (2001).

[33] V. M. Bedanov and F. M. Peeters, Phys. Rev. B 49, 2667 (1994).

[34] W. P. Ferreira, B. Partoens, F. M. Peeters, and 
G. A. Farias, Phys. Rev. E 71, 021501 (2005).

[35] R. Bubeck, P. Leiderer, and C. Bechinger, Progr. Colloid Polym. Sci. 118, 73 (2001).

[36] A. Melzer,, Phys. Rev. E 73, 056404 (2006).

[37] R. Bubeck, S. Neser, C. Bechinger, and P. Leiderer, Progr. Colloid Polym. Sci. 110, 41 (1998).

[38] V. M. Bedanov, G. V. Godiayak, and Yu. E. Lozovik, Zh. Eksp. Teor. Fiz. 88, 1622 (1984) [Sov. Phys. JETP 61,
967 (1985)]

[39] M. Bonitz, D. Block, O. Arp, V. Golubnychiy, H. Baumgartner, P. Ludwig, A. Piel, and A. Filinov, Phys. Rev. Lett. 96, 075001 (2006).

[40] The "zig-zag" transition takes place when the singlechain of particles splits into two chains. 